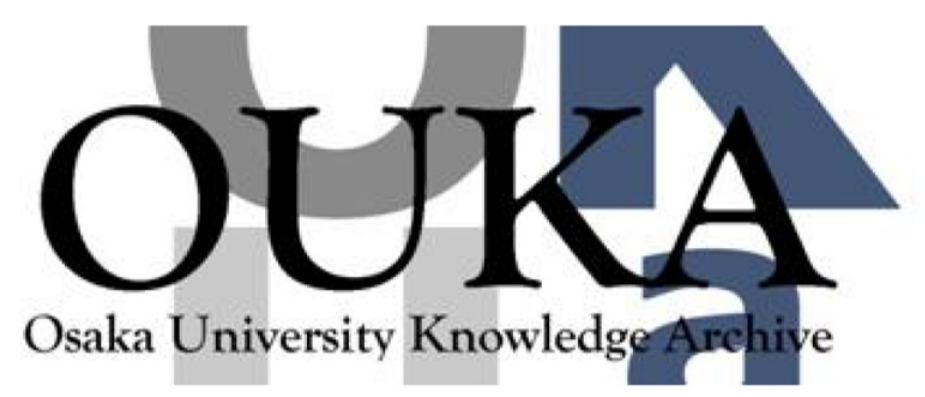

\begin{tabular}{|c|l|}
\hline Title & $\begin{array}{l}\text { Wearable Sanitizer: Design and Implementation } \\
\text { of an Open-source, On-body Sanitizer }\end{array}$ \\
\hline Author(s) & $\begin{array}{l}\text { Pataranutaporn, Pat; Shtarbanov, Ali; } \\
\text { Fernandes, Glenn et al. }\end{array}$ \\
\hline Citation & $\begin{array}{l}\text { SIGGRAPH Asia 2020 Emerging Technologies, SA } \\
\text { 2020. p. } 1\end{array}$ \\
\hline Issue Date & 2020-12-04 \\
\hline oaire:version AM \\
\hline URL & https://hdl. handle. net/11094/78269 \\
\hline rights & \\
\hline Note & \\
\hline
\end{tabular}

Osaka University Knowledge Archive : OUKA

https://ir. Library. osaka-u. ac. jp/

Osaka University 


\section{Wearable Sanitizer: Design and Implementation of an Open-source, On-body Sanitizer}

\author{
Pat Pataranutaporn \\ MIT Media Lab \\ Cambridge, MA, USA \\ Jingwen Li \\ MIT Media Lab \\ Cambridge, MA, USA
}

\author{
Ali Shtarbanov \\ MIT Media Lab \\ Cambridge, MA, USA \\ Parinya Punpongsanon \\ Osaka University \\ Toyonaka, Osaka, Japan \\ Pattie Maes \\ MIT Media Lab \\ Cambridge, MA, USA
}

\author{
Glenn Fernandes \\ MIT Media Lab \\ Cambridge, MA, USA \\ Joe Paradiso \\ MIT Media Lab \\ Cambridge, MA, USA
}
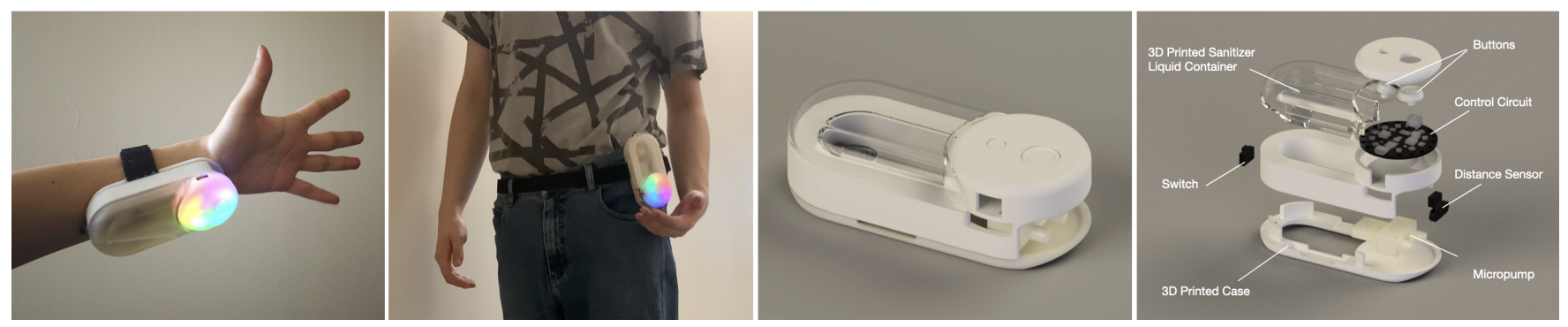

Figure 1: Wearable Sanitizer is a device that integrates with the user's body and behavior to automatically dispense alcohol onto their skin, nearby objects, and surfaces. The device body consists entirely of 3D printed parts and low-cost electronics.

\begin{abstract}
During the pandemic, wearables such as face masks and face shields have become broadly adopted, these solutions do reduce infection but do not eliminate infectious agents from surfaces and objects the person may touch. Therefore, regular disinfection of hands and frequently touched surfaces is a critical factor in preventing the spread of infectious diseases ranging from the common cold and flu to SARS and COVID-19. This activity of frequent disinfection requires a high degree of discipline and leads to increased cognitive and physical effort involved in frequent washing of hands or use of a pocket sanitizer. We present an open-source, wearable sanitizer that provides just-in-time, automatic dispensing of alcohol to the wearer's hand or nearby objects using sensors and programmable cues. We systematically explore the design space aiming to create a device that not only seamlessly integrates with the user's body and behavior but also frees their physical and mental faculties for other tasks.
\end{abstract}

Permission to make digital or hard copies of part or all of this work for personal or classroom use is granted without fee provided that copies are not made or distributed for profit or commercial advantage and that copies bear this notice and the full citation on the first page. Copyrights for third-party components of this work must be honored. For all other uses, contact the owner/author(s).

SA '20 Emerging Technologies, December 04-13, 2020, Virtual Event, Republic of Korea (C) 2020 Copyright held by the owner/author(s)

ACM ISBN 978-1-4503-8110-9/20/11.

https://doi.org/10.1145/3415255.3422897

\section{CCS CONCEPTS}

- Human-centered computing $\rightarrow$ Interaction devices.

\section{KEYWORDS}

Wearable Device, Interaction Design, Sensitization, Ubiquitous computing.

\section{ACM Reference Format:}

Pat Pataranutaporn, Ali Shtarbanov, Glenn Fernandes, Jingwen Li, Parinya Punpongsanon, Joe Paradiso, and Pattie Maes. 2020. Wearable Sanitizer: Design and Implementation of an Open-source, On-body Sanitizer. In SIGGRAPH Asia 2020 Emerging Technologies (SA '20 Emerging Technologies), December 04-13, 2020. ACM, New York, NY, USA, 2 pages. https://doi.org/10. $1145 / 3415255.3422897$

\section{INTRODUCTION}

Human-factors engineering researchers have a long history in leading the design and development of technologies that help people stay safe, secure resources for survival, manage information, and improve many facets of life [Vivacqua et al. 2016]. During the pandemic, a new standard of using face masks and face shields has been broadly adapted for protecting individuals from infectious agents such as viruses or bacteria [Armani et al. 2020]. However, these solutions do not eliminate infectious agents from objects and surfaces which people might come into contact with. Therefore, one of the highly recommended practices for the public during and post-pandemic is the frequent disinfection of hands and frequently 
accessed surfaces [CDC 2020]. If a person relies on a pocket sanitizer, they need to remember to use it after contact with objects and surfaces.

Our work offers an example of how the HCI research could help support the public during-and-post a critical situation. We worked on rapidly prototyping an open-source, wearable sanitizing device that can automatically dispense Isopropyl Alcohol (IPA) or standard hand sanitizer gel to disinfect the wearer's hand and nearby objects using low-cost sensors and programmable cues (Figure 1). Our goal for this device is to disinfect effectively while minimizing the amount of sanitizing products used by considering the type of interaction, local infection risk and user's behavior.

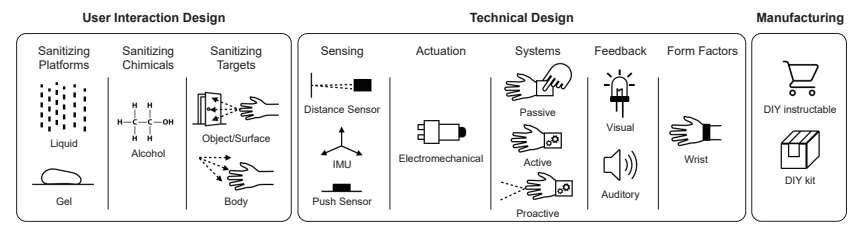

Figure 2: Wearable Sanitizer design space considers 3 main HCI factors: user interaction, technical design, manufacturing.

\section{DESIGN CONSIDERATION}

Our design space considers 3 main factors: the user interaction space, the technical design of the device and manufacturing considerations (Figure 2).

User Interaction Space. The user interaction space was derived from previous work that studied wearable hand hygiene to enhance hand wash frequency in a clinical environment [Boscart et al. 2008]. In this space, a user may either need to sanitize a specific target such as a doorknob or their own hand. Although designing a device to spray on a target would require knowledge of target surface geometry, using a simple distance sensor could at least inform the device about the target proximity. Depending on the method used, the sanitizing platforms could be divided into three categories: liquid, gel, UV. Exposure to frequent UV intensity can lead to health issues such as direct DNA damage. Hence, the use of sanitizing liquids is preferred.

Technical Design. A wearable sanitizer system could be passive, active, or proactive. A passive system dispenses the sanitizing medium using hand pressure, whereas an active one automatically dispenses the sanitizing liquids at the push of button. To free up the user's cognitive load, our proactive design includes automatic sensing and actuation based on the Inertial Measurement Unit (IMU) data for gesture recognition, and on Distance sensor data for approximation of the interaction space.

To pump a liquid from the device to the body or surface, we chose to use electro-mechanical actuation as a liquid pump because of its ease of use, small form factor, power efficiency and safety for use as a wearable device. Once the target is sprayed with the liquid, we need to indicate to the user that the target is safe to touch. For this feedback notification, we integrated both a visual method using
LEDs and an auditory feedback using a small speaker. The user can select either mode using the software or an embedded button.

The device itself could take multiple form factors. The most appropriate approach given our design decisions was to create a form factor that could be easily worn on the wrist. However, there could be other form factors that users could build themselves to fit personal usage based on different situations.

Manufacturing. One of our goal is creating a DIY kit and an instructable to enable everyone to build upon this platform ${ }^{1}$, and adapt it to different situations. Creating such a kit requires the availability of parts, ease of assembly as well as efficient assembly of the kit. Keeping these constraints in mind, we have built an effective device optimizing the sanitizing platform with ready-touse software.

\section{IMPLEMENTATION AND EVALUATION}

The hardware consists of a control board (Adafruit Circuit Playground), to which multiple components are connected, an alcohol reservoir that fits on the wrist to minimize the overall size, a laser distance sensor (TOF10120) to approximate the interaction distance between hand and surface, a $3 \mathrm{v}$ micropump with motor driver to control the amount of released alcohol, a Lithium-ion battery battery $(500 \mathrm{mAh})$, and an adjustable wrist strap. For mode control and user feedback, we used a switch, and RGB LEDs. We have also designed a 3D printable case to house the components, considering the ergonomics, size, and materials for rapid prototyping (Figure $1 \mathrm{~d})$. The dimensions of our device are $4 \mathrm{~cm} \times 5.5 \mathrm{~cm} \times 12.5 \mathrm{~cm}$. It takes approximately 2 hours to fully charge the device, and it lasts for at least 3 hours of use on a single charge.

Preliminary Evaluation. We conducted a short experiment to estimate the spraying characteristics needed to reach a target surface by varying parameters such as spraying period and Pulse Width Modulation (PWM) cycle which controls spraying velocity. We found that our wearable device can be used for sanitizing hands and surfaces of arbitrary shape up to $80 \mathrm{~cm}$ away. Since the spraying range and duration are controlled by the software, we can adopt this data in conjunction with the actual use case. We hope that our open-source design will help reduce the spread of infectious agents during the pandemic and beyond.

\section{REFERENCES}

2020. Cleaning and Disinfecting Your Facility. https://www.cdc.gov/coronavirus/2019ncov/community/disinfecting-building-facility.html

Andrea M Armani, Darrell E Hurt, Darryl Hwang, Meghan C McCarthy, and Alexis Scholtz. 2020. Low-tech solutions for the COVID-19 supply chain crisis. Nature Reviews Materials 5, 6 (2020), 403-406.

V.M. Boscart, K.S. McGilton, A. Levchenko, G. Hufton, P. Holliday, and G.R. Fernie. 2008. Acceptability of a wearable hand hygiene device with monitoring capabilities. fournal of Hospital Infection 70, 3 (2008), 216 - 222.

Adriana Vivacqua, Ana Cristina Garcia, José Canós, Martina Comes, and Vaninha Vieira. 2016. Collaboration and Decision Making in Crisis Situations. In Proceedings of the 19th ACM Conference on Computer Supported Cooperative Work and Social Computing Companion (San Francisco, California, USA) (CSCW'16 Companion). Association for Computing Machinery, New York, NY, USA, 503-508.

\footnotetext{
${ }^{1}$ see open-source: https://www.media.mit.edu/projects/wearable-sanitizer/
} 